\title{
Sub-barrier backward quasielastic scattering: A probe of the hexadecapole deformation
}

\author{
H.Q. Zhang, C.J. Lin, H.M. Jia, L. Yang, X.X. Xu, F. Yang, \\ Z.D. Wu, Z.H. LiU, N.R. MA, L.J. Sun and D.X. WAnG
}

China Institute of Atomic Energy, P. O. Box 275(10), Beijing 102413, P. R. China

\begin{abstract}
The quasielastic scattering excitation functions for ${ }^{16} \mathrm{O}+{ }^{152} \mathrm{Sm}$, ${ }^{170} \mathrm{Er}$ and ${ }^{174} \mathrm{Yb}$ were measured with high precision at a backward angle with small energy intervals at energies near the Coulomb barriers. The hexadecapole deformation $\left(\beta_{4}\right)$ of the target nuclei was extracted by using the lower-energy data and the obtained values agree with the available results reasonably well. This offers a sensitive method to extract $\beta_{4}$. This method is especially meaningful for the radioactive nuclei considering the low beam intensities.
\end{abstract}

\section{Introduction}

The role played by nuclear structure in near-barrier fusion reactions with heavy-ions has been observed several decades ago [1] and has been described well within the coupled-channels (CC) theoretical framework. Beside the quadrupole deformation $\left(\beta_{2}\right)$ effect, the possible role of the hexadecapole deformation $\left(\beta_{4}\right)$ in sub-barrier fusion has been frequently addressed $[2,3]$. The shape of the experimental fusion barrier distribution is equivalent to the theoretical result obtained by including classically random orientation of the deformed nucleus [4] and $\beta_{4}$ is expected to have a significant effect on fusion. The experimental fusion barrier distributions [5] mean that fusion reactions are very sensitive not only to $\beta_{2}$ but also to small $\beta_{4}$. 
Up to now, the methods of sub-barrier $\alpha$ scattering [6], above-barrier $\alpha$ excitation [6,7], electron scattering [8], and muonic $\mathrm{x}$ rays [9] have been used to determine the shape of the deformed nucleus experimentally. Usually, $\beta_{4}$ is difficult to extract experimentally and the obtained results are model-dependent and quite different with big errors. Theoretically, the macroscopic-microscopic method to calculate the ground-state deformation has been given in Ref. [10].

Based on the two complementary processes of fusion and backward quasielastic (QEL) scattering, we try to extract $\beta_{4}$ by using the lower-energy QEL data where the coupling effect to nuclear deformation is expected to be dominant. Also QEL is easier to be measured experimentally at lowerenergy region. To this end, the spherical ${ }^{16} \mathrm{O}$ was selected as projectile. The nuclei of ${ }^{152} \mathrm{Sm},{ }^{170} \mathrm{Er}$ and ${ }^{174} \mathrm{Yb}$, which can be seen as good rotors with similar $\beta_{2}$ but very different $\beta_{4}$ for the ground-state rotational band, were selected as target nuclei.

\section{Experimental procedure and data analysis}

The experiment was performed based on the HI-13 tandem accelerator at China Institute of Atomic Energy (CIAE). Thin targets $\left(50-100 \mu \mathrm{g} / \mathrm{cm}^{2}\right.$ thick) of ${ }^{152} \mathrm{Sm},{ }^{170} \mathrm{Er}$ and ${ }^{174} \mathrm{Yb}$ with a diameter of $3 \mathrm{~mm}$ evaporated onto $20-25 \mu \mathrm{g} / \mathrm{cm}^{2}$ carbon foils were used. The beam energy range is $E_{\mathrm{Lab}}=$ 40-80 MeV. The QEL particles were measured by using four silicon surface barrier detectors placed at the backward angle of $\theta_{\text {Lab }}=175^{\circ}$. Another group of four silicon surface barrier detectors were placed symmetrically at the forward angle $\theta_{\mathrm{Lab}}=41^{\circ}$ relative to the beam direction as monitor detectors and also for data normalization. More details can be found in [11].

The energy window for QEL was defined to include the elastic peak and the tail corresponding to all other peripheral processes. For all reactions, the QEL counts were divided by the sum of the elastic scattering counts in the four monitor detectors, giving a quantity proportional to the ratio of the QEL cross sections to Rutherford scattering $\mathrm{d} \sigma_{\mathrm{QEL}} / \mathrm{d} \sigma_{\mathrm{Ru}}$, where 'Ru' denotes Rutherford scattering. The measured experimental QEL excitation functions for ${ }^{16} \mathrm{O}+{ }^{152} \mathrm{Sm},{ }^{170} \mathrm{Er}$ and ${ }^{174} \mathrm{Yb}$ are shown in fig. 1 (left), where only statistical errors are given and the typical errors are smaller than $1 \%$.

An effective energy $E_{\text {eff }}$ was used by considering the minor centrifugal energy $E_{\text {cent }}$ correction, that is $E_{\text {eff }}=E_{\text {c.m. }}-E_{\text {cent }}$, in order to compare the barrier distribution $D_{\mathrm{QEL}}\left(E_{\mathrm{c} . \mathrm{m} .}, 175^{\circ}\right)$ with $D_{\mathrm{QEL}}\left(E_{\mathrm{eff}}, 180^{\circ}\right)$. The corresponding barrier distributions are shown in fig. 1 (right). Considering the 


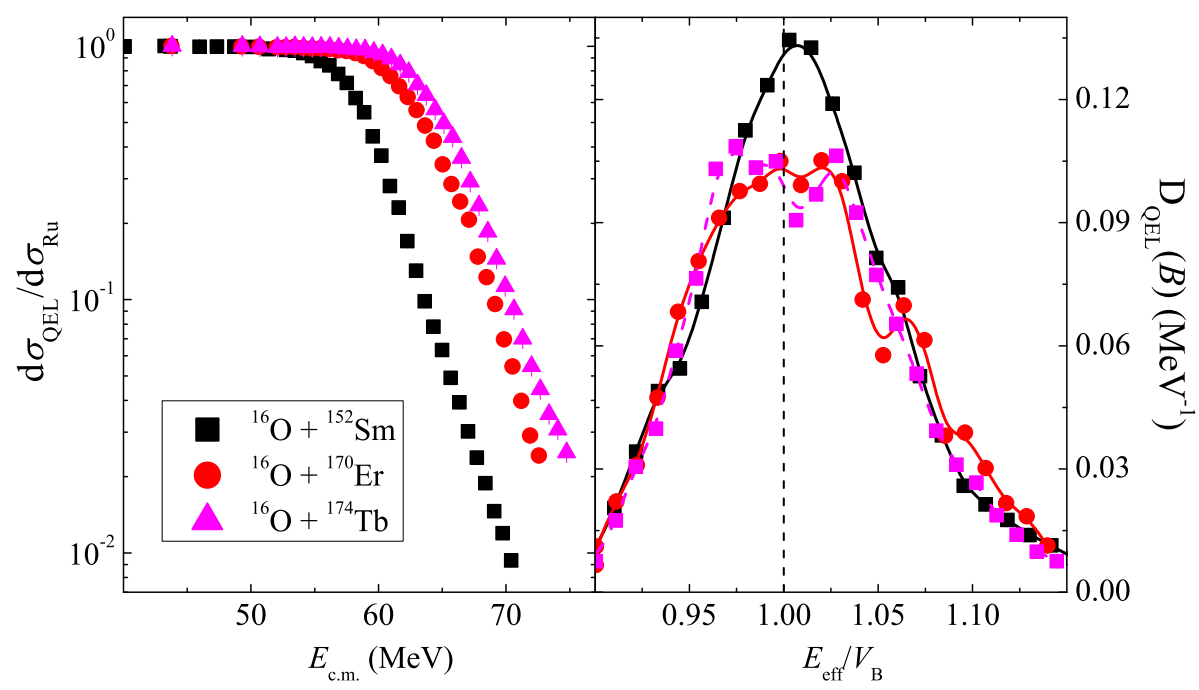

Figure 1: Experimental QEL excitation functions (left) for ${ }^{16} \mathrm{O}+{ }^{152} \mathrm{Sm},{ }^{170} \mathrm{Er}$ and ${ }^{174} \mathrm{Yb}$ measured at $\theta_{\mathrm{Lab}}=175^{\circ}$ and the extracted barrier distributions (right).

prevailing sensitivity of QEL to nuclear deformation at lower energies and to simplify the problem further by reducing the possible effect of other reaction channel couplings on the extracted $\beta_{4}$, only the data of $\mathrm{d} \sigma_{\mathrm{QEL}} / \mathrm{d} \sigma_{\mathrm{Ru}}>0.7$ was used in the following analysis somewhat arbitrarily.

The CC calculations were performed with a modified version of the code CCFULL [12]. A Woods-Saxon (WS) potential with $r_{0}=1.20 \mathrm{fm}$ and $a=$ $0.65 \mathrm{fm}$ was used, the potential depth $V$ was varied to reproduce the barrier heights. As usual, a short range imaginary potential with $W=30 \mathrm{MeV}$, $r_{0 W}=1.0 \mathrm{fm}$, and $a_{W}=0.4 \mathrm{fm}$ was used to simulate the compound nucleus formation. The used Coulomb radius parameter is $r_{0 \mathrm{C}}=1.1 \mathrm{fm}$, which has little influence on the calculated cross sections. The calculated results are insensitive to the parameters of the imaginary part of the potential as long as it is strong enough and well localized inside the Coulomb barrier.

Considering the coupling effect, the excitation energies of ${ }^{16} \mathrm{O}$ are high compared with the barrier curvature, so only produces an adiabatic potential renormalization, without affecting the structure of the barrier distribution [13]. This effect can be included in the potential and will not be considered explicitly in the CC calculations. Excitation states up to $10^{+}$in the rotational band of the target nuclei were included.

For ${ }^{16} \mathrm{O}+{ }^{152} \mathrm{Sm}$, both fusion and backward QEL have been studied 


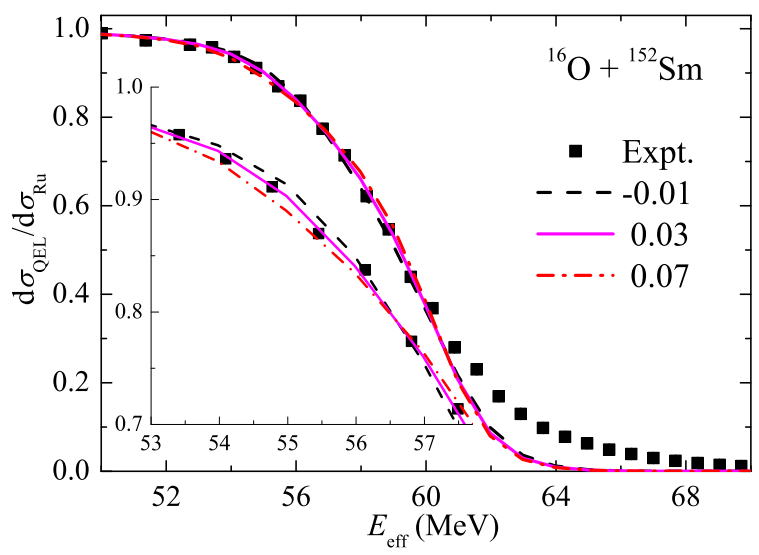

Figure 2: Experimental QEL excitation function for ${ }^{16} \mathrm{O}+{ }^{152} \mathrm{Sm}$ compared with the CC calculations by using different $\beta_{4}$.

widely [14]. Figure 2 shows the present-work backward QEL excitation function (solid squares). The lines are the $\mathrm{CC}$ calculations which take the permanent deformation of ${ }^{152} \mathrm{Sm}$ into account. $\beta_{2}=0.31$ [15] is used and fixed for ${ }^{152} \mathrm{Sm}$. Different $\beta_{4}$-values were used in the $\mathrm{CC}$ calculations for getting the optimal value by comparison with the experimental data. The parameter which gives the best reproduction for the lower-energy data well is $\beta_{4}=+0.037$ by using a chi-square analysis, the corresponding error is \pm 0.006 by making $\chi_{\min }^{2} / N_{\mathrm{pt}}=1.1$ for the optimal fit increased by 1 , where ' $N_{\mathrm{pt}}$ ' denotes the number of data points used in the fitting procedure. This means that the lower-energy backward QEL is indeed sensitive to higher order deformation $\beta_{4}$.

The extracted $\beta_{4}$-value for ${ }^{152} \mathrm{Sm}$ here is similar with both $\beta_{4}^{N}=0.038$ \pm 0.007 extracted from $14-18 \mathrm{MeV} \alpha$ scattering [16] and 0.048 obtained from $50 \mathrm{MeV} \alpha$ scattering [7], but smaller than both the charge deformation parameter of $\beta_{4}^{\mathrm{C}}=0.08$ [17] and $\beta_{4}=0.07\left(\beta_{2}=0.287\right)$ [8] obtained from electron scattering.

Similar analysis was also performed for ${ }^{16} \mathrm{O}+{ }^{170} \mathrm{Er}$ and ${ }^{16} \mathrm{O}+{ }^{174} \mathrm{Yb}$. The $\beta_{4}$ extracted in this work are shown in fig. 3 (solid circles). Figure 3 also represents some available results for $\mathrm{Sm}, \mathrm{Er}$ and $\mathrm{Yb}$ isotopes, which include the charge deformation $\beta_{4}^{\mathrm{C}}\left(\alpha^{\mathrm{C}}\right)$ extracted by means of the Coulomb excitation experiments $[6,17-19]$, nuclear deformation $\beta_{4}^{\mathrm{N}}\left(\alpha^{\mathrm{N}}\right)$ by using the interference effects [16] and inelastic scattering of $\alpha$ particles at energies well above the barrier $[6,7]$. Also included in this plot are the $\beta_{4}$ of ${ }^{152,154} \mathrm{Sm}$, 


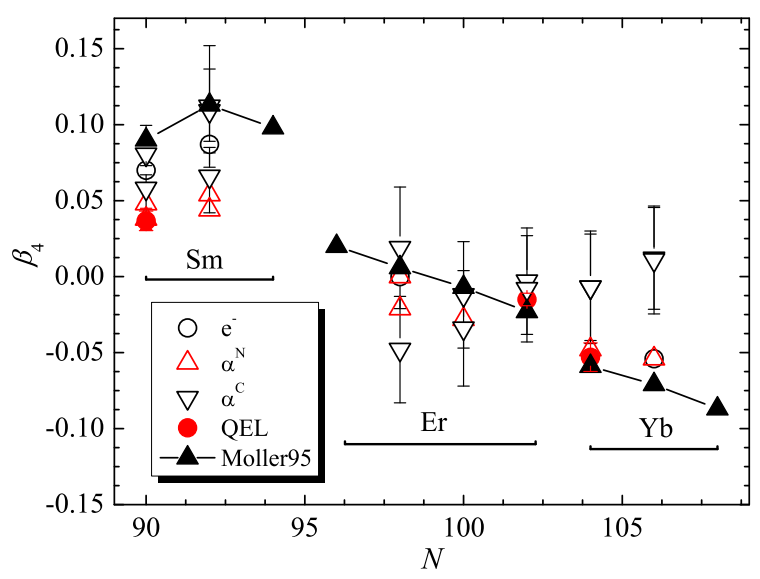

Figure 3: The variation of $\beta_{4}$ with neutron number $N$ for $\mathrm{Sm}$, Er and $\mathrm{Yb}$ isotopes by using different experimental methods and Möller's theoretical prediction [10].

${ }^{166} \mathrm{Er}$ and ${ }^{176} \mathrm{Yb}$ determined by electron scattering $\left(\mathrm{e}^{-}\right)[8]$. Deformations of the nuclear ground states calculated with a macroscopic-microscopic model (Möller95) [10] are also shown in fig. 3. It can be seen that the extracted $\beta_{4}$-values in this work qualitatively agree with this overall trend.

\section{Summary}

In summary, we have measured the QEL scattering excitation functions with high precision at a backward angle and extracted the barrier distributions for ${ }^{16} \mathrm{O}+{ }^{152} \mathrm{Sm},{ }^{170} \mathrm{Er}$ and ${ }^{174} \mathrm{Yb}$. The extracted $\beta_{4}$-values show a transition from positive for ${ }^{152} \mathrm{Sm}$ and near-zero for ${ }^{170} \mathrm{Er}$, to negative for ${ }^{174} \mathrm{Yb}$, which is consistent with the available trend. This means that the backward QEL at the lower-energy region, like fusion excitation function, is indeed sensitive to the higher-order deformation and especially the sign of $\beta_{4}$. Of course, more precise data and more systems are needed to further check the feasibility of this method. Comprehensive data analysis is also needed in order to get a more reliable quantitative result.

\section{Acknowledgements}

This presentation has been supported by the National Key Basic Research Development Program of China under Grant No. 2013CB834404, 
and the National Natural Science Foundation of China under Grants Nos. U1432246, U1432127, and 11505293.

\section{References}

[1] Stokstad R.G. et al., Phys. Rev. Lett., 41 (1978) 465.

[2] Rhoades-Brown M.J. and Oberacker V.E., Phys. Rev. Lett., 50 (1983) 1435.

[3] Dasgupta M. et al., Annu. Rev. Nucl. Part. Sci., 48 (1998) 401.

[4] Nagarajan M.A., Balantekin A.B. and Takigawa N., Phys. Rev. C, 34 (1986) 894.

[5] Lemmon R.C. et al., Phys. Lett. B, 316 (1993) 32.

[6] Lee I.Y. et al., Phys. Rev. C, 12 (1975) 1483.

[7] Hendrie D.L. et al., Phys. Lett. B, 26 (1968) 127.

[8] Cooper T. et al., Phys. Rev. C, 13 (1976) 1083.

[9] Powers R.J. et al., Phys. Rev. Lett., 34 (1975) 492.

[10] Möller P., At. Data Nucl. Data Tables, 59 (1995) 185.

[11] Jia H.M. et al., Phys. Rev. C, 90 (2014) 031601(R).

[12] Hagino K., Rowley N. and Kruppa A.T., Comput. Phys. Commun., 123 (1999) 143.

[13] Hagino K. et al., Phys. Rev. Lett., 79 (1997) 2014.

[14] Zhang Huanqiao et al., Phys. Rev. C, 57 (1998) R1047.

[15] Raman S., Nestor C.W. Jr., and Tikkanen P., At. Data Nucl. Data Tables, $\mathbf{7 8}$ (2001) 1.

[16] Brückner W. et al., Phys. Rev. Lett., 30 (1973) 57.

[17] Fischer H. et al., Phys. Rev. C, 15 (1977) 921.

[18] Erb K.A. et al., Phys. Rev. Lett., 29 (1972) 1010.

[19] Wollersheim H.J., Wilcke W. and Elze T.W., Phys. Rev. C, 11 (1975) 2008. 